\title{
Development of Obstacle Detection Shoes for Visually Impaired People
}

\author{
Chang-Min Yang, ${ }^{1}$ Ji-Yong Jung, ${ }^{2}$ and Jung-Ja Kim ${ }^{2,3 *}$ \\ ${ }^{1}$ Department of Healthcare Engineering, Jeonbuk National University \\ 567 Baekje-daero, Deokjin-gu, Jeonju-si, Jeollabuk-do 54896, Republic of Korea \\ ${ }^{2}$ Division of Biomedical Engineering, Jeonbuk National University, \\ 567 Baekje-daero, Deokjin-gu, Jeonju-si, Jeollabuk-do 54896, Republic of Korea \\ ${ }^{3}$ Research Center of Healthcare \& Welfare Instrument for the Aged, Jeonbuk National University, \\ 567 Baekje-daero, Deokjin-gu, Jeonju-si, Jeollabuk-do 54896, Republic of Korea
}

(Received June 10, 2019; accepted May 13, 2020)

Keywords: visually impaired people, obstacle detection shoes, walking assistive device, plantar pressure distribution, lower limb muscle activity

For visually impaired people, walking is very important in daily life because it provides independence and mobility. Visually impaired people typically use a white cane to assist them when walking, but it is difficult to detect low obstacles using a white cane. Therefore, many walking assistive devices for obstacle detection have been developed for visually impaired people. However, the gait characteristics of users have not been considered enough. Shoetype walking assistive devices have the advantage of detecting low obstacles and providing gait balance; however, no such devices have been developed yet. In this study, we developed obstacle detection shoes for visually impaired people, which can detect obstacles based on the foot angle. These obstacle detection shoes included infrared sensors, six-axis sensors, buzzers, and battery packs. The infrared sensors and six-axis sensors were attached to the upper part of the shoes to detect the distance of obstacles and the direction of the shoes. Additionally, the buzzers were placed on nearby ears to provide an alarm. All subjects were asked to walk along a corridor under two conditions: walking with a white cane and walking with the obstacle detection shoes. To evaluate the effectiveness of the developed device, the required time to pass, number of collisions, plantar pressure distribution, and lower limb muscle activity were analyzed during walking. We found that the required time to pass increased, while the number of collisions, peak pressure, and muscle activity decreased significantly when walking with the obstacle detection shoes. Consequently, we suggest that this device could be helpful in providing safety and supporting the stable walking of visually impaired people.

\section{Introduction}

Walking is a basic social activity that can improve physical and psychological health as well as life satisfaction. ${ }^{(1,2)}$ Individuals with visual impairment can only acquire nonvisual spatial information during walking. ${ }^{(3)}$ Accordingly, visually impaired people with limited information

${ }^{*}$ Corresponding author: e-mail: jungjakim@jbnu.ac.kr https://doi.org/10.18494/SAM.2020.2866 
may have more difficulty walking up and down stairs or negotiating complicated structures than other people. ${ }^{(4)}$

Independent ambulation plays a crucial role in the self-determination and gives confidence for individuals with visual impairment. Walking assistive devices have been used to avoid collision with unexpected obstacles during walking. The white cane is the most common assistive device owing to its advantages, such as reliability, simplicity, and low cost. ${ }^{(5,6)}$ However, the white cane also has several disadvantages including frequent collisions with physical objects for obstacle detection, ${ }^{(7,8)}$ difficulty in detection of obstacles located at low positions on the ground, and a lack of fall prevention caused by unnatural hand motion. ${ }^{(9)}$ In particular, visually impaired people tend to lose balance in even relatively small collisions and need more time to stand after collisions. ${ }^{(10,11)}$ Although walking with a guide dog is the preferred way to solve these problems, there are also some problems related to the high cost of training guide dogs and their limited use in indoor environments. ${ }^{(12)}$

Many walking assistive devices with improved functionality for obstacle detection and the latest navigation systems have been developed for visually impaired people. ${ }^{(13-15)}$ These devices can be classified into two different types: electronic canes and wearable walking assistive devices. Electronic canes, which are similar in usage to the conventional white cane, can provide a familiar method of use and comfort to blind persons. They can provide information such as the shape, dimension, and color of obstacles using specific sensors including an infrared laser and an ultrasonic sensor. Glasses or jackets are representative wearable walking assistive devices. They can be utilized to alert others to the fall of a user and detect a particular type of obstacle during walking. These types of devices are more efficient in minimizing dangers associated with falls than electronic canes. However, despite the advances in the technology of walking assistive devices providing accurate information, many devices are being developed without considering the gait pattern of users. To improve the accuracy of data from sensors attached to walking assistive devices, it is necessary to consider the walking direction of the user and the detection range of the sensor. Furthermore, the quantitative assessment of changes in biomechanical features characterizing gait is also essential when manufacturing walking assistive devices for individuals with visual impairment.

Therefore, the purpose of this study was to develop a new type of walking assistive device that can deliver accurate information by considering the walking direction and detection range of the sensor for obstacle detection. In addition, the required time to pass, number of collisions, plantar pressure distribution, and muscle activity in the lower limbs were compared to evaluate the effect of the developed device on the gait characteristics of visually impaired people during walking.

\section{Methods}

\subsection{Device design}

As shown in Fig. 1, the proposed walking assistive device, named as obstacle detection shoes, was composed of shoes, infrared distance sensors, Arduino 101 boards, buzzers, and 


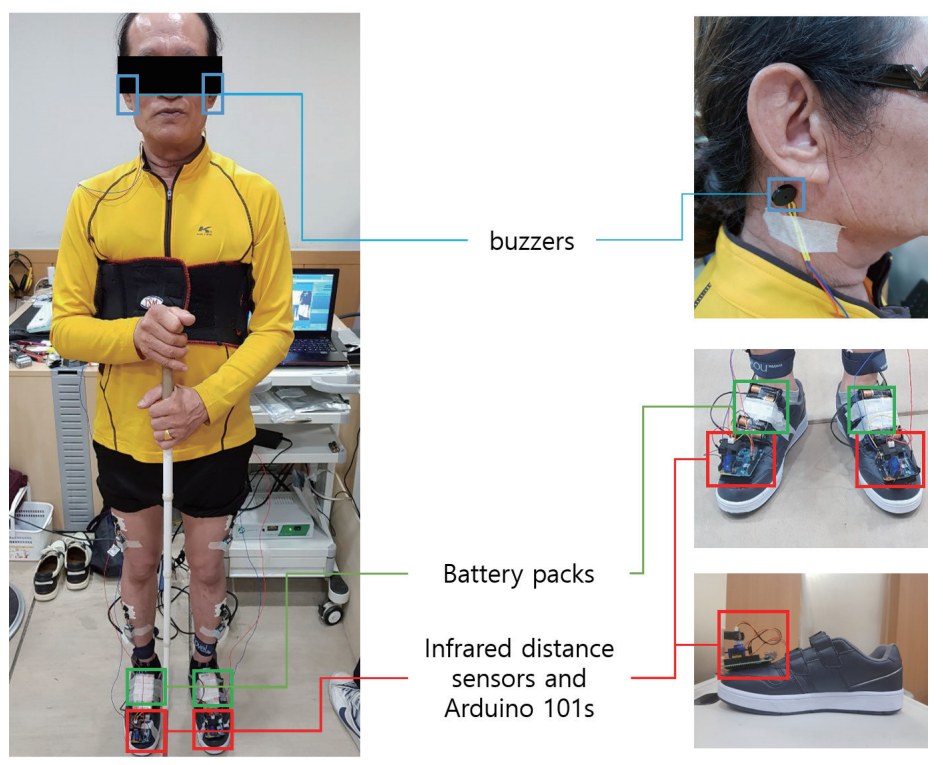

Fig. 1. (Color online) Obstacle detection shoes.

battery packs. The infrared sensors, Arduino 101 boards, and battery packs were all attached to the upper part of the shoes to detect the distance of obstacles while the buzzers were attached near the ear of the user to provide an alarm.

An ultrasound sensor, infrared sensor, and camera sensor have been most commonly used in previous walking assistive devices for visually impaired people to detect obstacles. An ultrasound sensor is able to minimize collisions by detecting over a wide range, while it is difficult to accurately determine the direction of obstacles. In contrast, when using an infrared sensor, it is possible to recognize an obstacle intuitively, while it has a narrow detection range. The camera sensor needs a high-performance microcontroller unit because of the large amount of data. In this study, an infrared sensor was utilized to distinguish obstacle locations intuitively using the foot angle during walking. To adjust the detection range of the sensor according to the walking direction, Arduino 101 boards, which contain Bluetooth, a six-axis accelerometer, and a gyrometer, were utilized. The direction of the infrared sensor was controlled with the six-axis data from the board, which corresponded to the foot angles during walking. The piezoelectric buzzer, which can make a beeping noise, was used to alert the user to the presence of obstacles as they walk along a corridor. It was designed to provide a dynamic alert sound to the user when there are obstacles within $60 \mathrm{~cm}$.

\subsection{Subjects}

Twelve adult men with visual impairment participated in this study. They were selected from the Visually Impaired Welfare Center in Jeonju, Republic of Korea. The mean age, height, and body weight of the subjects were $63.5 \pm 4.2$ years, $164.2 \pm 3.0 \mathrm{~cm}$, and $63.4 \pm 4.3 \mathrm{~kg}$, respectively. Subjects who had visual acuity of less than 0.04 were included in this study. 
Exclusion criteria were a history of injury in the musculoskeletal system, low-back pain, and gait abnormalities. All participants were given a full explanation of the protocol and provided written consent prior to their participation. This study was approved by the Institutional Review Board of Jeonbuk National University.

\subsection{Experimental setup}

All subjects were asked to walk along a corridor of $8 \mathrm{~m}$ length and $1.1 \mathrm{~m}$ width. This corridor was designed to have S-shaped and obstacle sections. All obstacles were made of plywood. As shown in Fig. 2, in the S-shaped section, obstacles were placed on the floor, forcing subjects to walk in an S-shaped trajectory. In the obstacle section, 12 obstacles were randomly placed on the floor. Experiments were divided into two conditions: (1) walking with a basic white cane with the obstacle detection shoes turned off and (2) walking with the obstacle detection shoes turned on without a white cane. The experiments were repeated three times each under the two conditions for each individual. In order to measure the effect of the walking assistive devices on the walking, the subjects performed each experiment wearing the same shoes. The required time to pass the obstacles, number of collisions, plantar pressure distribution, and muscle activity in the lower limbs were measured to determine the effect of the obstacle detection shoes on gait characteristics.

\subsection{Data analysis}

The required time to pass and number of collisions were assessed using LabVIEW 2010 software (National Instrument Corp, Texas, USA). A Pedar-X system (Novel GmbH, Munich, Germany) was used to measure the plantar pressure distribution during walking. Each insole of this system was composed of 99 capacitive sensors (sample rate $50 \mathrm{~Hz}$ ) and data were transmitted to a computer using a Bluetooth connection. All plantar pressure data were analyzed for peak pressure, which was divided into four regions: whole foot (WF), forefoot (FF),
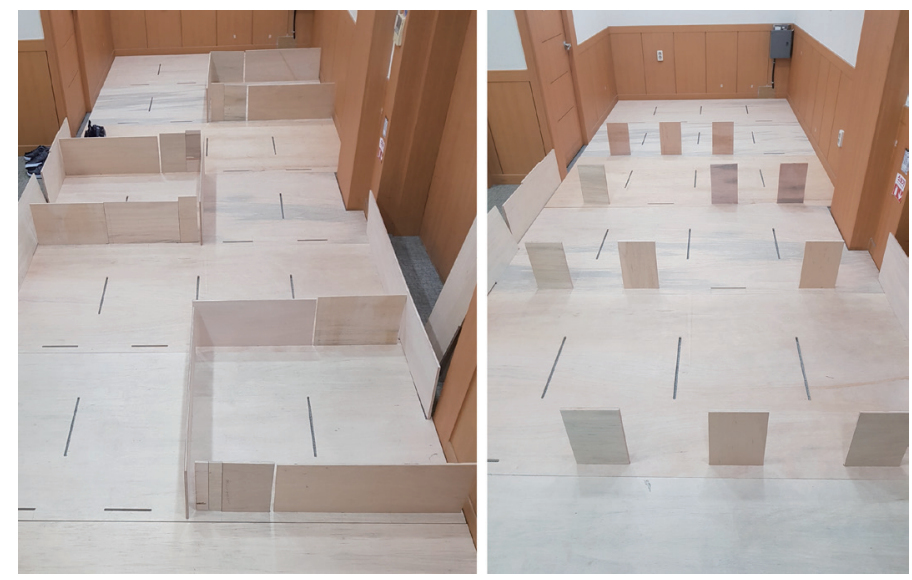

Fig. 2. (Color online) S-shaped and obstacle sections. 
midfoot (MF), and rearfoot (RF) regions. The surface electromyography (EMG) was recorded by utilizing a Noraxson Desktop DTS system (Noraxson Inc, Scottsdale, USA). The surface electrodes were attached to the vastas lateralis (VL), biceps femoris (BF), tibialis anterior (TA), and lateral gastrocnemius (LG) muscles on both sides. All EMG signals were amplified, bandpass-filtered (passband 20-450 Hz), and notch-filtered at $60 \mathrm{~Hz}$, with a sampling rate of $1000 \mathrm{~Hz}$. Data were expressed as a percentage relative to the reference voluntary contraction (RVC).

Statistical analysis was performed using SPSS 12.0 (IBM Corp., Chicago, USA). A normality Shapiro-Wilk test was conducted to determine the normality of all variables. Differences in the plantar pressure distribution and muscle activity between the left and right sides were compared by the Wilcoxon signed-rank test. In addition, the differences in all variables between walking with the white cane and walking with the obstacle detection shoes were analyzed through the Mann-Whitney $\mathrm{U}$ test. The significance level was set as $p<0.05$ and $p<0.01$.

\section{Results}

\subsection{Required time to pass and number of collisions}

Figures 3(a) and 3(b) respectively present the results of the average required time to pass and number of collisions in the S-shaped and obstacle sections. The required time to pass increased for both the S-shaped and obstacle sections when walking with the obstacle detection shoes compared with walking with the white cane. The number of collisions decreased in both sections when the obstacle detection shoes were used but the differences were not significant.

\subsection{Plantar pressure distribution}

Figure 4 shows the results of the plantar pressure distribution during walking. In the S-shaped section, the high peak pressure in all foot regions on both sides decreased significantly

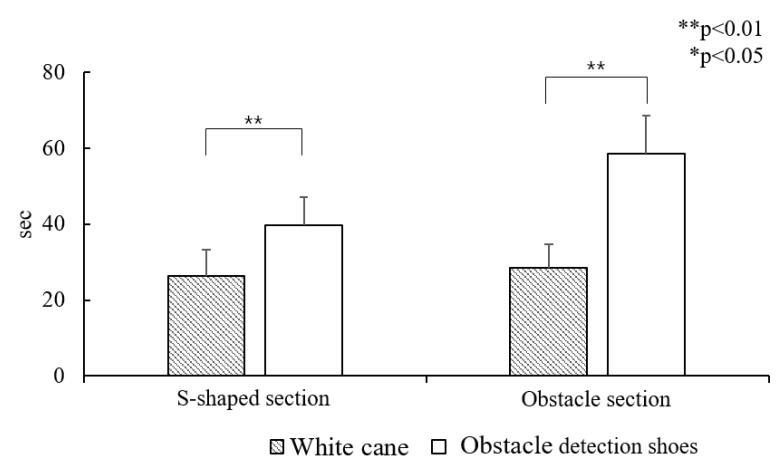

(a)

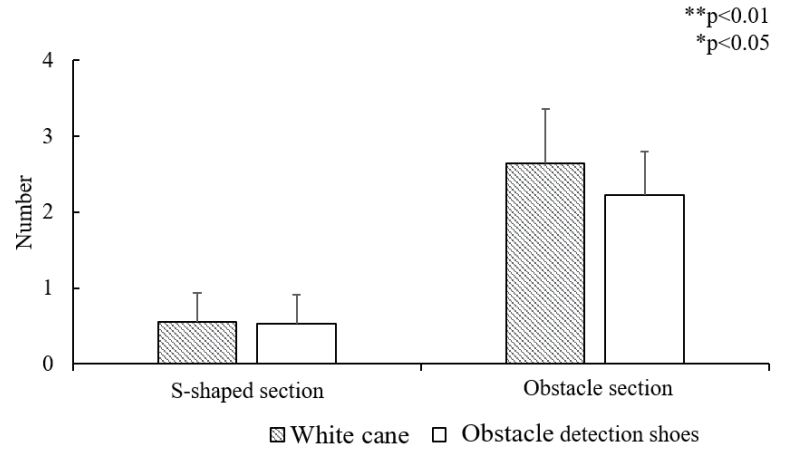

(b)

Fig. 3. (a) Required time to pass. (b) Number of collisions. 


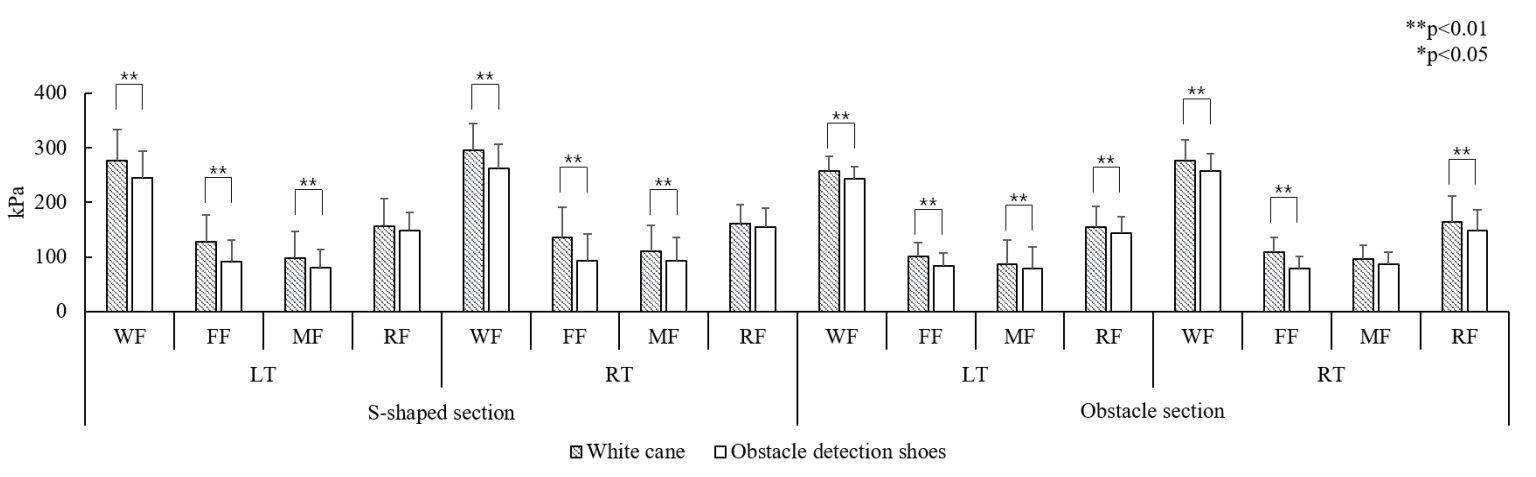

Fig. 4. Results of the plantar pressure distribution.

when walking with the obstacle detection shoes. On the left side, the peak pressure was reduced significantly by $11.52 \%(p=0.000), 27.99 \%(p=0.000)$, and $16.55 \%(p=0.000)$ in the WF, $\mathrm{FF}$, and MF, respectively. On the right side, it was reduced significantly by $11.21 \%(p=0.000)$, $31.02 \%(p=0.000)$, and $14.9 \%(p=0.000)$ in the WF, FF, and MF, respectively. Similar to the results in the S-shaped section, in the obstacle section, the high peak pressure in all foot regions on both sides also decreased significantly when walking with the obstacle detection shoes. On the left side, the peak pressure was reduced significantly by $5.85 \%(p=0.000), 17.16 \%(p=0.003)$, $11.17 \%(p=0.000)$, and $7.45 \%(p=0.007)$ in the WF, FF, MF, and RF, respectively. On the right side, it was reduced significantly by $6.8 \%(p=0.007), 28.05 \%(p=0.000)$, and $9.35 \%$ $(p=0.003)$ in the WF, FF, and RF, respectively. However, there were no significant differences in the peak pressure between the left and right sides under all experimental conditions.

\subsection{Muscle activity}

The results of the muscle activity are presented in Fig. 5. In the S-shaped section, all muscle activity was lower when walking with the obstacle detection shoes than when walking with the white cane. However, there was only a significant decrease in the activity of the TA muscle on the left side $(p=0.029)$. In addition, a significant difference in the muscle activity between the left and right sides was shown in the TA muscle when walking with the white cane $(p=0.032)$. In the obstacle section, the activity of all muscles decreased significantly when walking with the obstacle detection shoes. On the left side, it was reduced significantly by $15.08 \%(p=0.005)$, $13.43 \%(p=0.004)$, and $19.11 \%(p=0.000)$ in the $\mathrm{BF}, \mathrm{LG}$, and TA muscles, respectively. On the right side, it was reduced significantly by $9.92 \%(p=0.029), 18.79 \%(p=0.001), 15.72 \%$ $(p=0.000)$, and $14.50 \%(p=0.001)$ in the VL, BF, LG, and TA muscles, respectively.

\section{Discussion}

The walking assistive device proposed in this study was designed to improve detection accuracy for obstacles located at a low position on the ground by considering the sensing range 


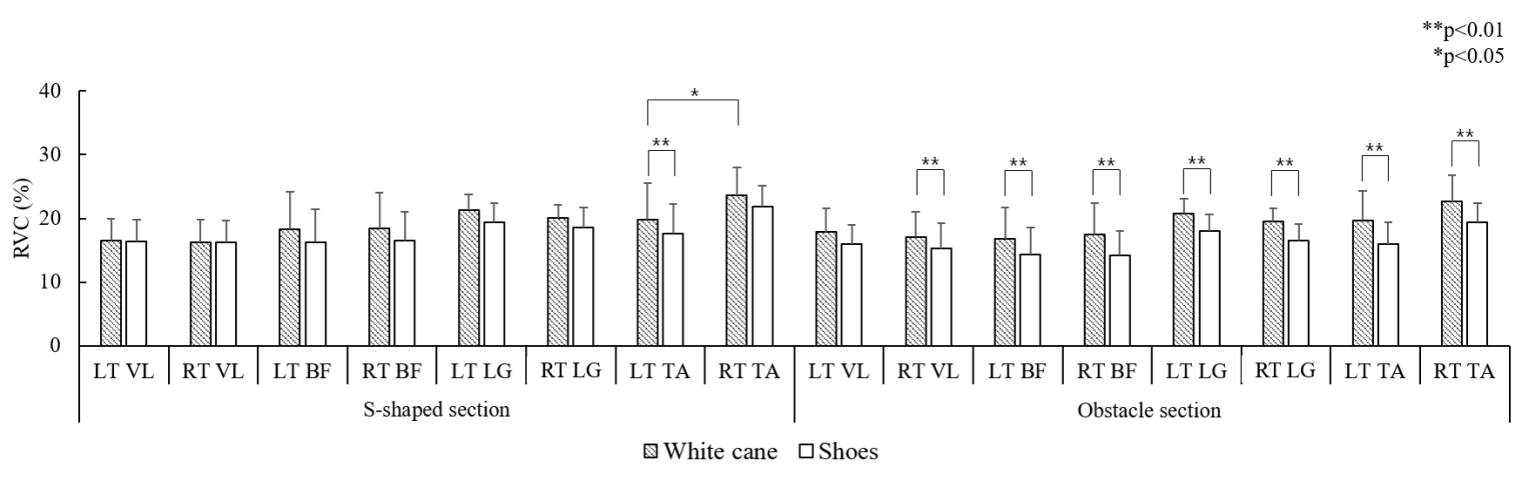

Fig. 5. Results of the muscle activity.

of the sensor and the user's walking direction. To evaluate the obstacle detection performance of the developed shoes, the required time to pass and the number of collisions were measured. In addition, plantar pressure distribution and lower limb muscle activity were measured to evaluate the effect on gait characteristics. The measured data were compared with those obtained when using a white cane, which is the most commonly used walking assistive device for visually impaired people. When comparing the results of all measured variables between the two experimental conditions, the required time to pass increased, while the number of collisions, plantar pressure distribution, and muscle activity all decreased when walking with the obstacle detection shoes.

The white cane is a simple walking assistive device that extends the tactile sensation of the user. Visually impaired people can acquire information about the location of the obstacle as well as the obstacle distance by direct contact during walking. In this study, a nondirect contact method was applied to the obstacle detection shoes to improve detection accuracy for obstacles located at a low position on the ground. It usually takes time for users to adapt to new types of walking assistive devices with sensors. Accordingly, the increased required time to pass when walking with the obstacle detection shoes may be due to the decreased gait speed because the subjects have not adapted enough to the device in our experiments. In the case of visually impaired people, the number of collisions may be associated with falls, causing various types of injury. In particular, decreasing the frequency of collisions is very important for elderly visually impaired people. ${ }^{(16)}$ Therefore, the result of this study, which shows a decrease in the number of collisions, is meaningful for developing walking assistive devices to prevent injuries of visually impaired people.

With respect to the plantar pressure distribution, the high peak pressure in all foot regions, including the WF, FF, MF, and RF, was reduced when walking with the obstacle detection shoes. The high peak pressure may be caused by the gait characteristic of visually impaired people, which has more forward propulsion than normal during walking while detecting obstacles. Previous research found that the reduction of plantar pressure is a critical component and key factor in the pathogenesis of plantar wounds in the elderly. ${ }^{(17)}$ This means that the use of the obstacle detection shoes should promote injury prevention by decreasing the peak pressure of elderly visually impaired people. ${ }^{(18-20)}$ 
There was decreased muscle activity when walking with the obstacle detection shoes. In particular, the decreased activity of all muscles in the lower limb when walking with the obstacle detection shoes may be correlated with the decreased gait speed indicated by the increased required time to pass. ${ }^{(21)}$ Low muscle activity may be related to the decreased muscle fatigue and improved walking condition of visually impaired people, which could reduce postural imbalance as well as prevent falls. ${ }^{(22)}$

Several walking assistive devices for visually impaired people were developed by utilizing useful technologies such as various types of sensors, information fusion, RFID, and AI. ${ }^{(23)}$ However, visually impaired people may not be able to buy devices because of their relatively low average income and the high price of the devices; the minimum price of walking assistive devices is currently roughly $\$ 500$. To solve this problem, our new walking assistive device for visually impaired people has a simple structure and a light weight, and it should be possible to manufacture it at a low cost. However, it has some technical limits of accuracy in distance detection. Therefore, in future research, it is necessary to improve system performance by combining navigation and RFID technology. ${ }^{(24-26)}$

\section{Conclusions}

In this study, we have developed a new type of walking assistive device that can provide improved obstacle detection accuracy and an efficient walking strategy for visually impaired people. The developed obstacle detection shoes have the following advantages: (1) decreased plantar pressure and lower limb muscle activity, reducing user fatigue when walking, (2) decreased imbalance between left and right in lower limbs when walking (it is considered that the imbalance of the body will be reduced by the smaller difference between the left and right during long-time use), and (3) improved low-obstacle detection performance. In the case of using a white cane, large obstacles such as pillars and walls are easy to detect, but low obstacles of less than $30 \mathrm{~cm}$ height are difficult to detect. However, most blind people are easily knocked down and injured by these low obstacles; thus, the obstacles used in our experiment were low obstacles of less than $30 \mathrm{~cm}$ height. To simplify the structure and reduce the cost of the proposed walking assistive device, we used only infrared distance sensors, Arduino 101 boards, buzzers, and battery packs when manufacturing the device, which achieved higher detection performance than a white cane in our experiments, enabling safe walking.

\section{Acknowledgments}

This work was supported by a National Research Foundation of Korea (NRF) grant funded by the Korea government (MSIT) (No. NRF-2019R1A2C1008454).

\section{References}

1 D. Nandini and K. R. Seeja: J. King Saud University Comput. Inf. Sci. 31 (2017) 385. https://doi.org/10.1016/ j.jksuci.2017.03.005

2 B. Siddhartha, A. P. Chavan, and B. V. Uma: Mater. Today: Proc. 5 (2018) 10665. https://doi.org/10.1016/ j.matpr.2017.12.344 
3 A. S. machado, G. D. Bombach, J. Duysens, and F. P. Carpes: Arch. Gerontol. Geriatr. Suppl. 63 (2016) 67. https://oi.org/10.1016/j.archger.2015.11.005

4 M. Gleeson, C. Sherrington, S. Lo, and L. Keay: Clin. Rehabil. 29 (2015) 244. https://doi. org $/ 10.1177 / 0269215514542636$

5 C. Pigeon, T. Li, G. Pradel, and C. Lamellet: Gait Posture 67 (2019) 43. https://doi.org/10.1016/ j.gaitpost.2018.09.018

6 P. Gharani, and H. A. Karimi: Image Vis. Comput. 64 (2017) 103. https://doi.org/10.1016/j.imavis.2017.06.002

7 E. S. Silva, G. Fischer, R. G. Rosa, P. Schons, L. B. T. Teixeira, W. Hoogkamer, and L. A. Peyre-Tartaruga: Gait Posture 62 (2018) 355. https://doi.org/10.1016/j.gaitpost.2018.03.049

8 D. Pascollini and S. P. Mariotti: Br. J. Ophthalmol. 96 (2012) 614. https://doi.org/10.1136/ bjophthalmol-2011-300539

9 R. B. Perreira, L. A. C. Grecco, and C. Oliveira: Gait Posture 57 (2017) 161. https://doi.org/10.1016/ j.gaitpost.2017.06.008

10 A. K. Buldt, J. J. Allan, K. B. Landorf, and H. B. Menz: Gait Posture 62 (2018) 56. https://doi.org/10.1016/ j.gaitpost.2018.02.026

11 Y. Ma, N. Amini, and H. Ghasemzadeh: Microprocess. Microsyst. 46 (2016) 67. https://doi.org/10.1016/ j.micpro.2016.07.001

12 C. L. Lee, C. Y. Chen, P. C. Sung, and S. Y. Lu: Appl. Ergon. 45 (2014) 817. https://doi.org/10.1016/ j.apergo.2013.10.012

13 D. Y. Jeong, J. Kwahk, S. H. Han, J. Park, M. Lee, and H. Jang: J. Transp. Health 10 (2018) 334. https://doi. org/10.1016/j.jth.2018.06.001

14 N. A. Howell, S. Farber, M. J. Widener, and G. L. Booth: J. Transp. Health 7 (2017) 160. https://doi.org/10.1016/ j.jth.2017.08.011

15 K. Y. Chan, U. Engelke, and N. Abhayasinhe: Expert Syst. Appl. 67 (2017) 272. https://doi.org/10.1016/ j.eswa.2016.09.007

16 A. Maeda, K. Nakamura, A. Otomo, S. Higuchi, and Y. Motohashi: Arch. Phys. Med. Rehabil. 79 (1998) 994. https://www.ncbi.nlm.nih.gov/pubmed/9710175

17 M. Bisiaux and P. Moretto: Gait Posture 28 (2008) 693. https://doi.org/10.1016/j.gaitpost.2008.05.009

18 S. Kasahara, H. Saito, T. Anjiki, and H. Osani: Gait Posture 42 (2015) 448. https://doi.org/10.1016/ j.gaitpost.2015.07.056

19 P. R. Cavanagh and S. A. Bus: J. Vasc. Surg. 52 (2010) 375. https://doi.org/10.1016/j.jvs.2010.06.007

20 K. Khanuja, J. Joki, G. Bachmann, and S. Cuccrullo: Maturitas 110 (2018) 51. https://doi.org/10.1016/ j.maturitas.2018.01.021

21 Z. Suica, J. Romkes, A. Tal, and C. Maguire: J. Bodyw. Mov. Ther. 20 (2016) 65. https://doi.org/10.1016/ j.jbmt.2015.06.002

22 T. Yan, M. Cempini, C. M. Oddo, and N. Vitello: Rob. Auton. Syst. 64 (2015) 120. https://doi.org/10.1016/ j.robot.2014.09.032

23 N. H. Cordeiro and E. C. Pedrino: Comput. Electron. Eng. 77 (2019) 61. https://doi.org/10.1016/ j.compeleceng.2019.05.003

24 W. Choi and S. Savarese: Computer Vision-ECCV 2010 (Springer, Heidelberg, 2010) 1st ed., Chap. 2.

25 A. Bugeau and P. Perez: Comput. Vis. Image Underst. 113 (2009) 459. https://doi.org/10.1016/j.cviu.2008.11.005

26 C. Stauffer and W. E. L. Grimson: Proc. IEEE Computer Society Conf. Computer Vision and Pattern Recognition (IEEE, 1999) 246-252.

\section{About the Authors}

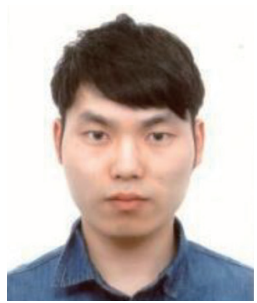

Chang-Min Yang is a Ph.D. student at the Department of Healthcare Engineering, Jeonbuk National University. His major research interests are wearable devices for the visually impaired, gait analysis, and functional shoes. (whycm@jbnu.ac.kr) 


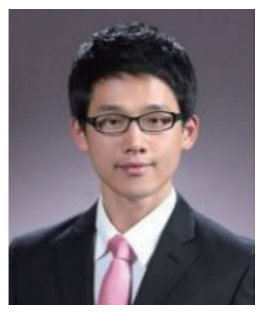

Ji-Yong Jung received his B.S., M.S., and Ph.D. degrees from Jeonbuk National University, South Korea, in 2010, 2012, and 2016, respectively. He is currently a research assistant professor at Jeonbuk National University. His major research interests are postural balance, gait analysis, and rehabilitation. (cholbun@hanmail.net)

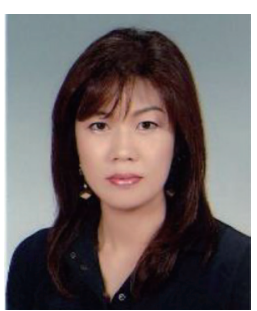

Jung-Ja Kim is a professor at the Department of Healthcare Engineering, Jeonbuk National University. Her major research interests are big data analysis (biological and medical data analysis), artificial intelligence, and rehabilitation. (jungjakim@jbnu.ac.kr) 